\title{
Perdurance, location and classical mereology
}

\section{HAROLD W. NOONAN}

In his (2001) Ted Sider takes care to define the notion of a temporal part and his doctrine of perdurantism using only the temporally indexed notion of parthood - ' $x$ is part of $y$ at $t$ ' - rather than the atemporal notion of classical mereology - ' $x$ is a part of $y^{\prime}-$ in order to forestall accusations of unintelligibility from his opponents. However, as he notes, endurantists do not necessarily reject the classical mereological notion as unintelligible. They allow that it makes sense and applies to atemporal subject matters and to temporal subject matters when the entities under discussion are not continuants. Thus they allow that it makes sense to say that metaphysics is a part of philosophy, or that football is a game of two halves. What (some) endurantists deny is only that the classical mereological notion is applicable to continuants: continuants (people, cats, statues etc.), they say, have no proper parts simpliciter (if we adopt the classical mereological definition we must say that they are atoms), either because it is false to say that they have or because it is unintelligible.

Thus perdurantists do not have to embrace Sider's excessive caution in defining their position. ${ }^{1}$ They can safely allow themselves classical mereological notions as long as it is a consequence of their definitions that continuants are perdurers/have temporal proper parts only if they have atemporal proper parts. $^{2}$

\footnotetext{
${ }^{1}$ And a reason for not doing so is a well-known difficulty with Sider's own definitions. It is controversial whether any continuant has a Siderian temporal part other than itself (a proper Siderian temporal part) since by his definition (2001: 59) if $x$ is a temporal part of $y$ at $t, x$ is a part of $y$ at $t$ and $x$ overlaps at $t$ any part of $y$ at $t$-so if $x$ is not $y x$ is a proper part of $y$ at $t$ yet $x$ is as big as $y$ at $t$ (the whole is not greater than the proper part). If we replace the clause ' $x$ is a part of $y$ at $t$ ' with the clause ' $y$ overlaps at $t$ any part of $x$ at $t$ ' this difficulty is avoided, but it becomes controversial whether any of a continuant's Siderean 'temporal parts' other than itself are parts at all (if it is denied that the atemporal notion of parthood applies to continuants).

${ }^{2}$ Of course, perdurantists could not safely define their position in this way if endurantists typically insisted both that it was unintelligible to apply classical mereological notions to continuants and that the thesis that continuants are perdurers was manifestly intelligible, albeit false, but such a combination of views is hardly that of the typical endurantist.
} 
In his (2006) Josh Parsons illuminatingly takes on the task he describes as 'get[tting] the allegedly technical (and, sometimes, allegedly incomprehensible ) concepts of temporal part, perdurance and so on by ratcheting up from mereological relations, subregion relations among times and the concept of exact temporal location (or alternatively, weak temporal location).' He continues, 'My definitions provide a good answer to those endurantists who claim that, though they can understand classical mereology, they cannot understand what a temporal part would be.

Moreover, since my definitions, and the theory of location as a whole, do not decide the question of endurantism versus perdurantism - and rightly so, I think, for it is not a question to be decided on conceptual grounds alone - these endurantists cannot claim that the question has been begged against them. ${ }^{3}$

I admire Parsons' work, but I think that a rearrangement of the pieces of his conceptual jigsaw, set out below, is more illuminating still. I have three reasons for thinking this. First, the locative concepts he puts forward as primitive in the quotation above are not in fact primitive in his scheme, as his glosses show, and it is illuminating to spell things out using the concept which is, in fact, primitive for him (or strictly, a concept which is immediately derivable from it). Secondly, Parsons does not in fact define the most basic notion of temporal part, ' $x$ is a temporal part of $y$ at $t$ ', but rather the concept 'the temporal part of $x$ at $t$ '. Thirdly, we shall see that the rearrangement allows much simpler definitions of the notions of perdurance and endurance than Parsons gives.

\footnotetext{
${ }^{3}$ A referee suggests that Parsons 'puts the cart before the horse'. But this could only be so if his primitive notion - that of a region's being completely free of a continuant (see below) - could only be understood if explained in terms of his target notion - that of a temporal part. But even if his primitive notion has to be explained before it can be understood, it can be explained, as the referee in fact indicates, using the endurantist-friendly notion of temporally indexed parthood: a time $t$ is completely free of an object $x$ if there is no object located at $t$ and a part of $x$ at $t$.
} 
Parsons glosses his notions of weak (temporal) location and exact (temporal) location in terms of the notion of a (temporal) region's being completely free of an object (as the parentheses indicate Parsons actually defines locative notions more generally and regards the temporal locative notions as a special case). An object is weakly located at a region if and only if the region is not completely free of it. It is exactly located at a region if and only if no part of the region is completely free of it and every disjoint region is completely free of it. He also defines what it is for an object to pervade a region: no part of the region is completely free of it.

Thus the twentieth century was not completely free of me, though its first half was (I was born in 1950), and nor is the twenty-first, though I expect its second half will be. So I did not pervade the twentieth century but I pervaded its second half and every decade therein. Again, 1914 was not completely free of the 1914-18 War, though the first months of 1914 were, nor was 1918, though its last days were. So the 1914-18 War did not pervade 1914, nor the years 1914-18, though it did pervade 1915-17 and each of those three years.

We can now use the notion of pervasive location together with the atemporal parthood relation of classical mereology to define perdurance, endurance and temporal parthood:

$x$ persists $=_{\mathrm{df}} x$ pervades two disjoint times ${ }^{4}$ $x$ perdures $=_{\mathrm{df}} x$ persists and $x$ has a proper part (which is at no time spatially smaller than it) ${ }^{5}$ $x$ endures $=_{\mathrm{df}} x$ persists and does not perdure

\footnotetext{
${ }^{4}$ A time is an instant or continuous or discontinuous interval.

${ }^{5}$ Is my head a proper part of me? It is a proper part of me now, and since we are never, I hope, going to go our separate ways, it is a proper part of me at any time it exists, but does that make it a proper part of me simpliciter? If the endurantist allows this (and thus abandons the hard line position that the classical mereological notion has no application to continuants) we need this parenthetical clause in our characterisation of perdurance. Ditto for the parenthetical clause in the following definition of ' $x$ is a temporal proper part of $y$ at $t^{\prime}$.
} 
$x$ is a temporal proper part $=_{\mathrm{df}} x$ is a temporal proper part of something $x$ is a temporal proper part of $y=_{\mathrm{df}} x$ is a temporal proper part of $y$ at some time $x$ is a temporal proper part of $y$ at $t=_{\mathrm{df}} x$ is a proper part of $y$ (and is at no time spatially smaller than $y$ ) and $x$ pervades $t$ and no time disjoint from $t$ $x$ is a temporal part of $y$ at $t=_{\mathrm{df}} x$ is a temporal proper part of $y$ at $t$ or $x=y$ and $y$ pervades $t$ and no time disjoint from $t$ $x$ is a temporal part of $y=_{\mathrm{df}} x$ is a temporal part of $y$ at some time $x$ is a temporal part $=_{\mathrm{df}} x$ is a temporal part of something.

We may now return to the beginning and define ' $x$ perdures', without the need for a parenthetical clause, as ' $x$ persists and $x$ has a temporal proper part'.

Of course, stronger notions are definable, for example, 'has a temporal part at every time it pervades'. Most of those who are self-labelled perdurantists, with the analogies between spatial and temporal extent in mind, will go beyond the contention that continuants are perdurers in the sense defined in the last paragraph. But they need not go so far as maintaining that they are perdurers in the strong sense defined at the beginning of this one. Most will hold, however, at least that continuants are perdurers in this intermediate sense: has a temporal part at every time interval it pervades. Conversely, not every self-labelled endurance theorist will take the hard line that continuants have no atemporal proper parts, and some will even allow that in some cases, e.g., of same origin temporary coincidence, a short-lived entity (a statue) may be an atemporal proper part of a longer-lived spatially equal-sized object (lump of clay). But endurance theorists will typically reject the intermediate strength perdurance doctrine. However, someone who makes the concessions just noted to the perdurantist and also concedes that for every time interval a continuant pervades there exists an atemporal proper part of it at no time spatially smaller than it which 
pervades that interval and no disjoint time - and thus qualifies as an intermediate strength perdurantist - may wish, as a referee suggests, still to think of himself as a (profligate) endurantist, either because he is still, after all, not committed to strong perdurantism, or because he has another notion of temporal part (what?) in accordance with which an intermediate strength perdurantist can deny that continuants have temporal parts. The important point is that, however we label them, using Parson's concepts we can define a variety of distinguishable positions ranging from hard-line endurantism (footnote 5), via perdurantism in the sense of the preceding paragraph, intermediate strength perdurantism (or profligate endurantism) and strong perdurantism, to the still stronger perdurantism which consists in the conjunction of strong perdurantism with Lewis's doctrine of the non-fundamentality of the temporally indexed. For, it is important to note, not every contentious issue is resolved once strong perdurantism is accepted. There can still be debate about Lewis's doctrine. Granted that I have a temporal part when I am bent, it does not follow, even given strong perdurantism, that that temporal part is bent simpliciter or even bent then; all that follows is that it is a proper part of something which is bent then. Without strong or at least intermediate perdurantism Lewis's solution to the problem of temporary intrinsics is unavailable; but it is not obligatory if these doctrines are taken on board.

In addition to the classical mereological notions and the notion of temporal location expressed by ' $x$ pervades $t$ ' (defined as 'no part of $t$ is completely free of $x$ ') the only notion used in the definitions given (in the parenthetical clauses) is that expressed by ' $x$ is spatially smaller than $y$ at $t$ '. This is understood as that relation which holds now between my head and myself, and also holds now between my wife and myself (following her recent diet). The endurantist cannot reject this notion. 
A question arises. Since on the account given processes and events, as well as continuants, have temporal proper parts if the perdurantist is right, what distinguishes them? Why are wars and warriors, lectures and lecturers, feasts (even solitary midnight feasts) and feasters, and football matches and footballers of distinct ontological categories? Well, football may be a game of two halves, but the first half of a football match is not itself a football match, whereas (ignoring the distinction between phase and substance sortals for the moment) as endurantists (all those who accept that continuants endure in the original sense defined in the text) would agree, every temporal part of a footballer is a footballer, Not ignoring the distinction between phase and substance sortals, we can say that if a substance sortal ' $F$ ' is a term for a type of continuant, every temporal part of an $F$ is an $F$. That is the distinction between continuant terms and event-or-process terms and therewith between continuants and events-or-processes themselves.

University of Nottingham

Nottingham, NG72RD, UK h.w.noonan@nottingham.ac.uk

\section{References}

Parsons J. 2006 Theories of location. Oxford Studies in Metaphysics Vol. 3. Oxford: Oxford University Press

Sider T. 2001 Four-dimensionalism. Oxford: Oxford University Press 